$68 \mid 2006$

Fictions biographiques et arts visuels, $\mathrm{XIX}^{\mathrm{e}}-\mathrm{XX \textrm {e } ^ { \mathrm { e } }}$ siècles

\title{
Discours critique et fiction biographique dans les Portraits imaginaires de Pater et les Vies imaginaires de Schwob
}

\section{Agathe Salha}

\section{OpenEdition}

\section{Journals}

Édition électronique

URL : http://journals.openedition.org/recherchestravaux/129

DOI : 10.4000/recherchestravaux.129

ISSN : 1969-6434

Éditeur

UGA Éditions/Université Grenoble Alpes

Édition imprimée

Date de publication : 15 avril 2006

Pagination : 29-39

ISBN : 2-9518254-8-X

ISSN : 0151-1874

\section{Référence électronique}

Agathe Salha, « Discours critique et fiction biographique dans les Portraits imaginaires de Pater et les Vies imaginaires de Schwob», Recherches \& Travaux [En ligne], 68 | 2006, mis en ligne le 06 novembre 2008, consulté le 08 septembre 2020. URL : http://journals.openedition.org/recherchestravaux/129 ; DOI : https://doi.org/10.4000/recherchestravaux.129 
Agathe SALHA

Université Stendhal-Grenoble 3

\section{Discours critique et fiction biographique dans les Portraits imaginaires de Pater et les Vies imaginaires de Schwob}

En 1887 paraissent Imaginary Portraits de l'écrivain et critique d'art anglais Walter Pater. Ce recueil de quatre récits biographiques mêle des personnages authentiques comme le peintre Antoine Watteau et des figures imaginaires comme Denys l'Auxerrois ou Sébastian Van Storck. En 1896, Marcel Schwob publie les Vies imaginaires, recueil de biographies fictives consacrées à des êtres authentifiés par la tradition historique ou littéraire.

Les critiques ont souvent rapproché ces deux textes tout en opposant la démarche de leurs auteurs. Ainsi pour George Trembley, dans Marcel Schwob faussaire de la nature, les personnages de Pater seraient l'incarnation d'idées abstraites et, "sous le déguisement de la biographie ", l'auteur ferait en réalité " de la critique esthétique ». Watteau symboliserait donc la peinture "dans son essence éternelle " alors que Schwob chercherait à montrer dans les Vies imaginaires "le visage unique de Paolo Uccello ${ }^{1}$ ". Autrement dit, et pour reprendre les termes de la préface des Vies imaginaires, les Portraits de Pater illustreraient plutôt le point de vue généralisant de l'histoire, alors que la démarche biographique de Schwob serait axée sur la recherche du particulier et de l'individuel. Si tout sépare l'intention des deux auteurs d'après Georges Trembley, on trouve ailleurs l'idée d'un lien parodique entre leurs textes. Anne Henry affirme ainsi, dans la préface de sa traduction française des Essais sur l'art et la Renaissance de Pater :

1. G. Trembley, Marcel Schwob faussaire de la nature, Genève-Paris, Librairie Droz, 1969, p. 104. 
Quant à Marcel Schwob [...], il s'est contenté de pasticher avec esprit dans ses Vies imaginaires les Portraits imaginaires dans lesquels Pater fixe sa définition de la subjectivité par les seules idiosyncrasies, les singularités d'un caractère ${ }^{2}$.

On tentera ici d'inscrire la comparaison dans une perspective plus large et de montrer comment, à travers la fiction biographique, ces deux auteurs témoignent d'un moment de l'évolution de la réception et de l'interprétation des œuvres, et de l'activité critique. À une époque où l'histoire de l'art s'est constituée en discipline scientifique en se séparant de la tradition littéraire, Pater cherche à dépasser l'approche érudite et revendique une critique intuitive ou imaginative. Les Vies imaginaires de Schwob procèdent, semble-t-il, d'un même mouvement, mais elles le dépassent et le radicalisent : proposant la biographie imaginaire ou rêvée comme la réelle expression de la poésie originelle de l'artiste, elles marginalisent l'œuvre et la réduisent à un symptôme voire à un épiphénomène.

\section{De la critique à la fiction : Imaginary Portraits et Studies in Art and Poetry de Walter Pater}

Le recueil des Portraits s'ouvre sur une vie de Watteau, intitulée «A prince of court painters ${ }^{3}$ ». Cette vie est singulière à plus d'un titre. Il s'agit d'abord du seul texte du recueil consacré à un peintre et ainsi manifestement lié à l'œuvre critique et historique de Walter Pater, en particulier aux Studies in Art and Poetry. La biographie de Watteau est également la seule à mettre en scène un personnage réel, les trois autres figures étant imaginaires. Ces deux éléments, comme la place liminaire du portrait de Watteau, incitent donc à l'interpréter comme un texte de transition qui permet de mieux cerner les rapports entre histoire et fiction dans l'œuvre de Pater. L'invention est très limitée dans ce portrait qui n'évoque que des personnages ou des événements

2. "Walter Pater ou le plaisir esthétique ", préface de A. Henry dans Walter Pater, Essais sur l'art et la Renaissance, Paris, Klincksieck, 1985, textes présentés et traduits par A. Henry, p. 15-16. L'ouvrage ne reprend que partiellement l'essai de Pater dont il ne retient que les chapitres les plus significatifs. Le titre anglais du recueil est : The Renaissance, Studies in Art and Poetry. Cet ouvrage ainsi que Imaginary Portraits a fait l'objet d'une réédition à laquelle nous nous référerons désormais pour les citations en anglais (Walter Pater, Three major texts The Renaissance, Appreciations and Imaginary Portraits -, New York and London, New York University Press, 1986, edited with an Introduction by William E. Buckler.)

3. "Un prince des peintres de cour» dans la traduction de Philippe Neel que nous citerons désormais (Portraits imaginaires, Christian Bourgois éditeur, 1985, avant-propos de P. Neel, postface de Mario Praz). 
authentifiés par les biographes contemporains de Watteau ${ }^{4}$. Le passage à la fiction est uniquement marqué par le recours à un narrateur distinct de l'auteur. Le texte se présente en effet sous la forme d'un journal fictif écrit par une narratrice anonyme que l'on identifie aisément comme la sœur de JeanBaptiste Pater, lui-même peintre et disciple de Watteau et par ailleurs ancêtre supposé de Walter Pater. La fonction autobiographique du journal intime est cependant démentie par la chronologie interne du texte qui se limite strictement à la biographie de Watteau puisqu'il commence en septembre 1701, au moment où le Watteau entre comme apprenti chez le père de la narratrice, et s'achève à la mort du peintre, en 1721. La voix de la diariste imaginaire ne survit donc pas à son unique fonction : l'exploration passionnée du mystère de Watteau et de sa peinture.

Ce journal fictif évoque une œuvre et un personnage absents ou visibles par intermittences et une relation à sens unique puisque la narratrice, secrètement amoureuse de Watteau, est constamment ignorée par lui. La fiction revendiquée par le titre, Imaginary Portraits, ne donne jamais directement accès au monde intérieur du peintre; elle consiste uniquement en une mise en scène du regard du spectateur ou du critique symboliquement incarné par la narratrice amoureuse. Cette interprétation est confirmée par le point de vue anachronique de la narratrice dont les jugements sur la peinture de Watteau évoquent la tradition littéraire française du XIX siècle, en particulier l'essai des frères Goncourt sur l'art du XVIII siècle, paru en $1856^{5}$. Elle souligne, par exemple, le caractère profondément mélancolique de cette peinture, qui dévoilerait la grâce d'un monde aristocratique brillant, mais fragile et menacé. Elle y décèle également le pressentiment d'une époque révolutionnaire, marquée par une nouvelle ère « de fraternité, de liberté, d'humanité, d'une espèce inconnue de liberté sociale ${ }^{6}{ }^{\prime}$. Dans les dernières pages de son journal, peu avant la mort du peintre, elle livre en ces termes la clé, la formule, de la vie et de l'œuvre de Watteau :

4. Voir Vies anciennes de Watteau, textes réunis par Pierre Rosenberg, Hermann, 1984, ou l'ouvrage plus ancien de Pierre Champion, Notes critiques sur les vies anciennes d'Antoine Watteau, Champion, 1921.

5. E. et J. de Goncourt, L'Art du XVIIT siècle et autres textes sur l'art réunis et présentés par J.-P. Bouillon, Hermann, 1967. Sur la réception de l'œuvre de Watteau au XIX ${ }^{\mathrm{e}}$ siècle, voir en particulier, D. Posnar, "Watteau mélancolique : la formation d'un mythe », Bulletin de la société de l'histoire de l'art français (1974), p. 345-361 et J. Shirley, "Vues sur Cythère : Watteau et la critique romantique du XIX siècle ", Revue des Sciences humaines, ${ }^{\circ} 157$ (1975), p. 5-21.

6. "Un prince des peintres de cour", op. cit., p. 43-44. 
Il ne surmontera jamais l'influence de ses premières années, et toutes ces choses légères conserveront toujours, à ses yeux, une sorte de valeur représentative ou empruntée, et caractériseront ce monde impossible ou défendu que le fils du maçon contemplait à travers les portes closes du jardin enchanté. Ces grâces futiles et mièvres, symboles à ses yeux d'un noble monde d'aspirations et d'idées, ressuscitent pour lui, par le pouvoir de l'association, - et même maintenant qu'il en a compris la vraie insignifiance, - toute la vieille griserie magique de son rêve, de son rêve d'un monde meilleur que le monde réel ${ }^{7}$.

Le regard aimant de la narratrice remonte à la source, à l'intention originelle et découvre dans l'œuvre un sens qui échappe à son créateur lui-même. La contemplation est donc pleinement une création : elle révèle la vision intérieure de l'artiste et donne accès à sa conscience qui paraît marquée par une forme d'obsession et de transformation onirique de la réalité. La biographie apparait ainsi comme la forme naturelle de cette critique intuitive et imaginative.

Le portrait imaginaire de Watteau montre donc les apports spécifiques de la biographie d'une part et de la fiction d'autre part comme contribuant à l'expression d'une nouvelle forme critique : la biographie introduit le lecteur dans un espace de proximité avec l'artiste ; quant à la fiction, entièrement cristallisée sur la figure de la narratrice, elle permet de s'affranchir des contraintes de l'objectivité et de projeter une libre interprétation de sa vie intérieure. La vision de la narratrice évoque d'ailleurs celle de Pater luimême, étant largement anachronique par rapport à la réception de Watteau au XVIII siècle. Cet anachronisme explique que Walter Pater s'évade finalement vers les vies imaginaires car, comme le souligne Mario Praz dans la postface de l'édition française du recueil, «À la longue, le personnage historique se révèle incapable de supporter le contenu dont il [l'auteur] a voulu l'investir et qui a pourtant été à ses yeux la chose la plus importante ${ }^{8}$ ». Les autres portraits font donc surgir des personnages imaginaires de la contemplation d'un vitrail ou d'une série de tableaux par le narrateur. Toutes les fictions biographiques de Pater exhibent ainsi leur origine critique et montrent comment la projection de l'imaginaire du spectateur dans l'œuvre d'art fait surgir une vie rêvée de l'artiste.

7. Ibid. p. 45. ["He will never overcome his early training; and these light things will possess for him always a kind of representative or borrowed worth, as characterising that impossible or forbidden world which the mason's boy saw through the closed gateways of the enchanted garden. Those trifling and petty graces, the insignia to him of that nobler world of aspiration and idea, even now that he is aware, as I conceive, of their true littleness, bring back to him, by the power of association, all the old magical exhilaration of his dream - his dream of a better world than the real one. Imaginary Portraits, op. cit., p. 255 et 256.]

8. Op. cit., p. 209. 
Ce mélange de figures authentiques et imaginaires illustre l'ambiguïté profonde du portrait littéraire. Sainte-Beuve, qui revendique l'invention de cette nouvelle forme, en souligne d'ailleurs le caractère hybride. "Contrairement à l'opinion répandue ", écrit-il en 1844 dans Portraits de femmes, "la critique n'y occupe qu'une place secondaire " et le portrait est davantage un moyen pour " produire nos propres sentiments sur le monde et sur la vie, pour exhaler avec détour une certaine poésie cachée ». Mais s'ils permettent "de continuer l'élégie interrompue ", les Portraits s'apparentent également au genre romanesque et à la fiction puisqu'ils choisissent de préférence " des noms peu connus ou déjà oubliés " et forment ainsi "de petites nouvelles à un seul personnage ${ }^{9}$ ". Cette dissolution de la critique dans l'essai personnel et dans la fiction évoque exactement les Portraits imaginaires de Walter Pater. Elle s'amorçait déjà nettement dans ses essais historiques et critiques antérieurs consacrés aux artistes et aux poètes de la Renaissance française et italienne ${ }^{10}$.

Publié pour la première fois en 1873 , le recueil de Pater intitulé The Renaissance, Studies in Art and Poetry regroupe plusieurs textes consacrés à des peintres et à des sculpteurs italiens des quinzième et seizième siècles comme Léonard de Vinci, Michel-Ange ou Botticelli. Ces textes sont à la fois des commentaires et des réécritures des Vies de Vasari auquel Pater se réfere d'ailleurs explicitement dans les premières lignes de l'essai sur Léonard de Vinci :

9. "Madame de Charrière ", Portraits de femmes dans CEuvres de Sainte-Beuve, Gallimard, "Pléiade ", 1951, texte présenté et annoté par M. Leroy, p. 1353. Sur "l'invention " du portrait littéraire par Sainte-Beuve, voir également l'introduction de G. Antoine aux Portraits lit téraires de Sainte-Beuve (Robert Laffont, "Bouquins ", 1993), en particulier, "Une nouveauté : le portrait littéraire " (p. LXII à LXXIII).

10. Ce rapprochement entre les portraits de Sainte-Beuve et les essais critiques de Pater est confirmé par plusieurs allusions de ce dernier au critique français dans son ouvrage sur la Renaissance. Dans la préface de l'ouvrage en 1873, Pater le présente comme " a recent critique » et le cite en français, comme s'étant fixé le même but que lui dans son ouvrage : «De se borner à connaître de près les belles choses et à s'en nourrir en exquis amateurs, en humanistes accomplis " (The Renaissance, op. cit., p. 72). Pater cite encore Sainte-Beuve à plusieurs reprises dans l'étude qu'il consacre à Du Bellay dans le même ouvrage : "This impress M. de Sainte-Beuve thought he found in the Antiquités de Rome and the Regrets, which he ranks as what been called poésie intime, that intensely modern sort of poetry in which the writer has for his aim the portraiture of his most intimate moods and to take the reader into his confidence" (ibid., p. 180). [Cette empreinte que monsieur de Sainte-Beuve pense avoir trouvée dans les Antiquités de Rome et dans les Regrets, qu'il classe au rang de ce que l'on a appelé poésie intime, cette forme de poésie intensément moderne dans laquelle l'auteur se donne pour but de dépeindre ses états d'âme les plus intimes et de faire du lecteur son confident.] Nous traduisons. 
Sa " légende ", comme on dit en France, toute remplie d'anecdotes célèbres, a fourni à Vasari un de ses plus brillants chapitres. Les écrivains postérieurs se sont contentés de la recopier jusqu'à ce qu'en 1804 Carlo Amoretti l'ait soumise à une critique qui a ébranlé toute sa datation et qui n'a épargné aucun détail. Les diverses questions qui ont alors surgi ont fait l'objet depuis ce temps d'études spécialisées, et la simple érudition n'a plus grand-chose à faire. [...] Mais l'amateur d'âmes étranges peut toujours analyser pour lui-même l'impression que lui font ses œuvres, et tenter ainsi de définir les éléments essentiels de son génie ${ }^{11}$.

L'idéal critique ici défini est celui de l'amateur et du passionné, du curieux qui perçoit dans l'œuvre "l'intuition d'un secret ${ }^{12}$ ", la porte qui doit donner accès au génie du peintre. Ainsi, contrairement aux exigences de l'érudition, il importe peu à Pater de distinguer l'œuvre du maître lui-même de celle d'un élève qui aurait subi son influence. Il s'agit davantage pour lui, en s'appuyant sur les éléments figuratifs du tableau, de reconnaître le sentiment qui s'incarne dans l'œuvre, d'identifier une forme particulière de conscience, de dégager une individualité. Cette critique impressionniste et psychologique se présente comme l'aboutissement d'une double tradition puisqu'elle s'appuie sur les acquis de l'érudition moderne et sur la littérature biographique. Pater ajoute en effet que la "légende " pourra « de temps à autre intervenir pour corroborer les résultats de l'analyse ». Revendiquant un procédé déjà implicitement utilisé par Vasari, il assume donc l'invention biographique dans la mesure où elle contribue à la compréhension des œuvres.

La biographie de Vinci illustre bien cette double polarité de la vie et de l'œuvre, de la légende et du tableau. Les premières pages introduisent ainsi l'image traditionnelle de l'artiste assimilé à un mage et à un voyant :

Son idéal de beauté est si exotique qu'il nous fascine davantage qu'il nous séduit : plus que chez tout autre artiste, cet idéal semble refléter les pensées et presque les catégories d'un monde intérieur. Aussi ses contemporains le croyaient-ils détenteur d'un savoir caché, de source suspecte, tandis que Michelet et d'autres ont vu en lui un précurseur. Il a fait peu de cas de son propre génie et n'a produit ses chefs-d'œuvre que dans ses dernières années, si tourmentées ; pourtant, ce génie le tient si bien qu'il a pu traverser sans s'émouvoir les événements les plus tra-

11. Traduction d'A. Henry, op. cit., p. 74. ["His legend, as the French say, with the anecdotes which every one remembers, is one of the most brilliant chapters of Vasari. Later writers merely copied it, until in 1804, Carlo Amoretti applied to it a criticism which left hardly a date fixed, and not one of those anecdotes untouched. The various questions thus raised have since that time become, one after another, subjects of special study, and mere antiquarianism has in this direction little more to do. [...] But a lover of strange souls may still analyse for himself the impression made on him by those works, and try to reach through it a definition of the chief elements of Leonardo's genius.», op. cit., p. 135.]

12. J'emprunte cette expression et les analyses qui suivent à la préface d'Anne Henry ("Walter Pater ou le plaisir esthétique », op. cit., p. 37-38.) 
giques qui accablaient sa patrie et ses amis ; c'est comme s'il les croisait par hasard au cours de sa mission secrète ${ }^{13}$.

La première partie du texte de Pater est une mise en forme biographique de cette image romantique, ordonnée dans la perspective des chefs-d'œuvre ultimes dont la description occupe la fin de l'essai. Pater mêle au récit de la jeunesse et des errances du peintre à travers l'Italie, des traits psychologiques divers qui se font écho tout en reprenant certains lieux communs de l'esthétique romantique. La personnalité de Vinci est ainsi marquée par l'insatisfaction, le goût de l'étrange, les recherches désordonnées dans tous les domaines du savoir ; le peintre est obsédé par certaines images comme le sourire des femmes ou le mouvement des eaux où se joignent la beauté et la terreur ; son œuvre exprime enfin "l'idéal du beau auquel se mêle inextricablement un élément de dérision ". Dans cette première partie de la vie du peintre, Pater accumule les anecdotes invraisemblables sans jamais les accompagner d'un commentaire explicatif. Il accentue ainsi la fantaisie des Vies de Vasari déjà marquées par l'ellipse et la rupture, le goût de l'étrange et du romanesque. L'errance hallucinée du peintre culmine lors de son arrivée à Milan qui est décrite comme un rêve, mais cet épisode fantastique et dont le caractère fictif est évident livre en même temps la clé de l'univers intérieur et de l'art de Vinci :

Curiosité, désir de beauté - les voilà bien, les deux forces élémentaires du génie de Léonard : une curiosité souvent en conflit avec son désir de beauté, mais capable d'engendrer, quand ils s'unissent, un modèle de grâce étrange et subtile. Le mouvement qui anime le quinzième siècle est double [...] Raphaël représente le retour à l'Antiquité, Léonard celui à la nature. Et par ce dernier, il cherchait à satisfaire une curiosité infinie pour les surprises perpétuelles que cette nature pouvait lui offrir $[\ldots]^{14}$.

13. Op. cit., p. 73-74. ["His type of beauty is so exotic that it fascinates a larger number than it delights, and seems more than that of any other artist to reflect ideas and views and some scheme of the world within ; so that he seemed to his contemporaries to be the possessor of some unsanctified and secret wisdom ; as to Michelet and others to have anticipated modern ideas. He trifles with his genius, and crowds all his chief work into a few tormented years of later life; yet he is so possessed by his genius that he passes unmoved through the most tragic events, overwhelming his country and friends, like one who comes across them by chance on some secret errand. ", op. cit., p. 134-135.]

14. Op. cit., p. 80. [Curiosity and the desire of beauty - these are the two elementary forces in Leonardo's genius ; curiosity often in conflict with the desire of beauty, but generating, in union with it, a type of subtle and curious grace./The movement of the fifteenth century was two-fold [...]. Raphael represents the return to antiquity, and Leonardo the return to nature. In this return to nature, he was seeking to satisfy a boundless curiosity by her perpetual surprises [...]. Op. cit., p. 140-141]. 
Par un curieux retournement, la singularité du peintre devient emblématique de la Renaissance. C'est que la contradiction intime de Léonard traduit en réalité une appréciation esthétique de son œuvre et le catalogue qui suit le récit biographique confirme ce phénomène de miroir entre la vie et l'œuvre. Le travail d'écriture de Pater consiste donc à reprendre les éléments épars de la tradition et à les réordonner à la lumière d'une tension intérieure de Vinci, elle-même emblématique d'une idée générale puisqu'elle révèle la continuité entre la Renaissance et l'époque moderne. Il n'y a donc pas lieu d'opposer ces deux objectifs de Walter Pater dans son essai sur Vinci : mettre en valeur la singularité de l'artiste et les idiosyncrasies du génie et donner à sa vie une dimension exemplaire et historique. Si l'accumulation de traits divers et variés s'ordonne autour d'un centre qui donne sens et exemplarité à la vie de l'artiste, Pater souligne que cette formule, cette clé de l'artiste, naît de la seule intuition du regard critique qui s'apparente à une sorte de divination.

De Studies in Art and Poetry à Imaginary portraits, Pater tente de résoudre l'opposition entre critique érudite et critique imaginative, en les subordonnant à la valeur absolue de l'expérience esthétique. Ce point de vue subjectiviste autorise le critique à s'appuyer sur la légende de l'artiste et inspire un jeu dialectique avec la distance historique qui sépare l'œuvre de son interprète. Cette démarche annonce celle de Schwob dans les Vies imaginaires qui projette également son imaginaire sur la tradition et fait surgir la fiction d'une méditation sur l'histoire. Mais Schwob dépasse Pater et radicalise sa vision de la critique imaginative. Les Vies imaginaires sont donc moins un pastiche, au sens formel de ce mot, qu'une interprétation littérale et une mise en œuvre systématique des présupposés de l'historien anglais.

\section{Vies imaginaires de Marcel Schwob}

Comme les études de Pater sur les peintres de la Renaissance, la Vie de Paolo Uccello est une réécriture de Vasari. Schwob mentionne d'ailleurs sa source, ce qui est rare dans les Vies imaginaires, et il lui emprunte l'essentiel de ses anecdotes. Mais cette fidélité apparente masque une déformation importante du texte initial et de son propos psychologique et historique. Pour Vasari en effet, Uccello illustre l'exemple récurrent de l'artiste victime d'une chimère, ici les recherches dans le domaine de la perspective, et dont le talent est finalement gâché par son obsession. Cependant, la description précise des œuvres du peintre et la conclusion de la biographie montrent bien que, malgré l'échec personnel d'Uccello, ses recherches sur la perspective ont contribué aux progrès de son art. La réécriture de Schwob modifie 
radicalement ce propos en adoptant implicitement la vision romantique de l'artiste telle qu'elle apparaît notamment dans les fictions de peintres au XIX siècle. Il semble en fait que Schwob réécrive Vasari à travers le prisme déformant du Chef-d'euvre inconnu de Balzac selon un jeu de superposition des sources typique des Vies imaginaires et qui permet de faire flotter les destins individuels entre plusieurs époques historiques. Ainsi, les recherches sur la perspective, présentée comme un progrès technique indispensable à l'imitation du réel par Vasari, traduisent chez Schwob une ambition démiurgique : Uccello se détourne de l'imitation et, comme Frenhofer, le héros de Balzac, il cherche à découvrir le secret de la création à travers celui de la forme. À la fin de la nouvelle, Uccello dévoile à son ami Donatello une toile représentant saint Thomas : alors que le sculpteur n'y perçoit qu'un " fouillis de lignes » et conseille à Uccello de cacher sa toile, le peintre fou se méprend sur le sens de ses paroles croyant " qu'il a [avait] accompli le miracle ${ }^{15}$ ». La figure de saint Thomas, dénoncé dans les Évangiles parce qu'il a besoin de voir pour croire, symbolise implicitement celle de Donatello qui n'a peutêtre pas su voir le chef-d'œuvre du peintre. Mais cette erreur de jugement est curieusement redoublée par celle d'Uccello sur le sens de ses paroles. Cette ambiguïté évoque la fin de la nouvelle de Balzac qui s'achève également sur le dévoilement et l'échec du chef-d'œuvre de Frenhofer.

La biographie d'Uccello est ainsi entièrement construite sur le thème du secret : Schwob décrit d'abord le peintre caché au fond de sa maison et cherchant comme un alchimiste à percer les arcanes de la nature. Plus loin, Uccello est comparé à un ermite : "Ainsi vivait l'Oiseau, et sa tête pensive était enveloppée dans sa cape ; et il ne s'apercevait ni de ce qu'il mangeait ni de ce qu'il buvait, mais il était entièrement pareil à un ermite ${ }^{16}$ ". Quant au chef-d'œuvre rêvé, inachevé ou invisible, il est investi d'un pouvoir immense au plan de la fiction puisque tout l'univers du peintre, depuis ses richesses jusqu'à sa maison et sa femme, lui est progressivement sacrifié. Ce fil conducteur du secret ou du trésor caché montre que la Vie de Paolo Uccello est centrée, comme la nouvelle de Balzac, autour d'un même phénomène de non-réception de l'œuvre d'art. L'aveuglement de Donatello, ami de Paolo Uccello et premier spectateur de son œuvre, suggère que la proximité et la familiarité avec l'œuvre et même avec l'artiste ne garantissent pas l'accès à la vérité et à la beauté cachées. Au contraire, c'est la tâche du biographe de découvrir, par le biais de la fiction, l'intuition originelle et la source dont les

15. "Paolo Uccello, peintre " dans M. Schwob, Vies imaginaires, Gallimard, "L'Imaginaire », 1957, p. 101.

16. Ibid., p. 99. 
œuvres ne sont que des étapes ou des traces. Si Paolo Uccello se définit mieux par ce qu'il a voulu faire que par ce qu'il a fait, son rêve ou sa chimère donnent donc la vraie perspective sur son œuvre et sur sa vie.

Dans cette perspective, la Vie des peintres et des artistes n'est plus subordonnée à l'évocation de leur production, mais se confond avec l'œuvre chimérique qui figure une sorte de destin. Schwob supprime ainsi le catalogue descriptif des œuvres du peintre à l'exception de quelques tableaux mentionnés au début et à la fin de la nouvelle. L'élément descriptif traditionnel des Vies d'artistes est en fait directement intégré à la narration à travers d'innombrables allusions au cercle et à la ligne qui désignent l'obsession d'Uccello. Le peintre semble vivre dans son œuvre, selon le procédé que Schwob utilise aussi dans les vies d'écrivains comme Pétrone ou Cyril Tourneur.

La Vie de Paolo Uccello se comprend finalement par rapport à l'ensemble plus vaste des Vies imaginaires d'artistes, de philosophes ou d'écrivains qui parsèment le recueil de Schwob. Placées en tête de l'ouvrage, les Vies d'Empédocle et de Cratès exploitent un phénomène comparable de dévalorisation ou de marginalisation de l'œuvre par rapport à la vie de l'artiste. Le pur magicien et le philosophe cynique dont l'enseignement se limite à de rares maximes illustrent l'idéal antique d'une philosophie incarnée et d'une sagesse vécue dont l'éclat se passe du témoignage d'une œuvre. Si la Vie du thaumaturge grec est manifestement écrite sur le modèle de celle de Jésus, on peut voir de même en Cratès une sorte de Socrate radicalisé. Or, Socrate et Jésus, sources inspiratrices de la tradition occidentale que revisitent les Vies imaginaires, n'ont jamais rien écrit. Ces deux figures invisibles mais dont l'ombre plane sur l'ensemble du recueil de Schwob justifient implicitement l'art du biographe pour qui la vraie poésie est dans la vie des hommes.

Dans la préface des Vies imaginaires, Schwob définit l'art du biographe par la recherche de l'individuel et du particulier. Il affirme par exemple que la forme du nez ou les manies vestimentaires de Socrate l'intéressent plus que " ses interrogatoires de morale » et que "l'idéal du biographe serait de différencier infiniment l'aspect de deux philosophes qui ont inventé à peu près la même métaphysique ${ }^{17}$ ". Cette apologie provocatrice du détail et de l'idiosyncrasie masque en fait un déplacement plus essentiel de l'interprétation critique des œuvres vers une tentative d'appréhension plus intuitive d'une

17. Ibid., p. 11-12. 
vie spirituelle, d'une intention poétique ou philosophique. La fiction biographique apparait alors comme un nouveau genre hybride qui permet de saisir cette intention. De Pater à Schwob, l'œuvre se subordonne progressivement à la vie de l'artiste et à son monde intérieur. Ultimement, cette nouvelle forme de critique qu'est la fiction biographique peut tenter de s'affranchir de l'univers des artistes et des œuvres. Elle peut explorer de nouveaux mondes poétiques, ceux des hommes d'action qui n'écrivirent pas : Vies d'incendiaires, Vies de pirates, Vies d'assassins.

On soulignera, pour conclure, le paradoxe des Vies imaginaires, œuvre érudite, nourrie de la tradition artistique et littéraire, mais qui illustre la fascination de l'expérience vécue, et promeut un idéal d'action et d'aventure. Patient compilateur enfermé dans sa bibliothèque, le biographe rêve d'évasion à travers des vies de pirates et de criminels. Cependant, au fur et à mesure du recueil, on voit se multiplier les figures d'aventuriers ou de pirates sous influence qui n'agissent que par l'impulsion de fantasmes livresques. Le modèle de Don Quichotte court ainsi tout au long du livre et accentue la nostalgie d'une impossible évasion. Les Vies de Schwob s'inscrivent ainsi pleinement dans l'imaginaire littéraire de la fin-de-siècle qui multiplie ces libérations impossibles : départ avorté de des Esseintes pour l'Angleterre dans À Rebours, réincarnation de Durtal dans la figure de Gilles de Rais, grand soldat et grand criminel du Moyen Âge ou, plus modestement, embardée banlieusarde du narrateur de Paludes. On comptera au nombre de ces tentatives le voyage de Schwob presque agonisant aux îles Samoa, sur les traces de Stevenson. 
